\title{
NF-kB mediated CX3CL1 activation in the dorsal root ganglion contributes to the maintenance of neuropathic pain induced in adult male Sprague Dawley rats ${ }^{1}$
}

\author{
Chenglong Liu',Fei Zhang",Haihua Liu"','Fang Wei"
}

'MD, Associate chief Physician, Department of Anaesthesiology, Gaoyou People's Hospital, Gaoyou Hospital Affiliated, Soochow University, China. Conception and design of the study, manuscript preparation, final approval.

"MD, Physician, Department of Anaesthesiology, Gaoyou People's Hospital, Gaoyou Hospital Affiliated, Soochow University, China. Acquisition, analysis and interpretation of data; manuscript preparation.

I'MD, Physician, Department of Neurology, Gaoyou People's Hospital, Gaoyou Hospital Affiliated, Soochow University, China.Technical procedures, acquisition of data.

IVMD, Physician, Department of Radiology, Gaoyou People's Hospital, Gaoyou Hospital Affiliated, Soochow University, China. Analysis and interpretation of data, critical revision.

\begin{abstract}
Purpose: To evaluate the role of $\mathrm{CX} 3 \mathrm{CL} 1$ and NF-KB in the lumbar disc herniation induced neuropathic pain.

Methods: After LDH induced by implantation of autologous nucleus pulposus (NP) on the left L5 nerve root was established, mechanical thresholds and thermal hyperalgesia were tested at relevant time points during an observation period of 28 days. Expression of CX3CL1 and NF-kBin the dorsal root ganglion (DRG) were performed by using Western blotting and RTPCR.

Results: Implantation of autologous nucleus pulposus (NP) induced neuropathic pain, associated with increased mRNA and protein expression of CX3CL1 in the DRG. Moreover, intrathecal injection of neutralizing antibody against CX3CL1 could attenuates LDH-induced persistent pain hypersensitivity. Interestingly, NF-KB activation in the DRGs were found in LDH-induced neuropathic pain. Furthermore, NF-KB downregulation by p65 inhibitor PDTC markedly alleviated LDH-induced mechanical allodynia and thermal hyperalgesia in rat. Importantly, CX3CL1 neutralizing antibody $(10 \mu \mathrm{g} / 10 \mu \mathrm{l}$, i.t.) reduces p-p65 protein level in DRG

Conclusions: CX3XL1 could regulate LDH-induced neuropathic pain through NF-KB pathway. Targeting CX3CL1 and NF-KB may represent a potential treatment for neuropathic pain caused by LDH.
\end{abstract}

Key words: Neuralgia. Chemokine CX3CL1. NF-kappa B. Ganglia, Spinal. Rats. 


\section{Introduction}

Nowadays, Long-term chronic pain compromises the patients' life quality seriously. The disc herniation associated sciatica, which is the most common types of neuropathic pain, is a common cause of low back pain and sciatica. It is mainly caused by both mechanical lumbar nerve root deformation and local inflammation induced resulting from nucleus pulposus (NP) ${ }^{1-}$

3. Although previous studies suggested that various factors, including hereditary and age, might contribute to $\mathrm{LDH}$-induced neuropathic pain; the pathophysiology cause for LDHinduced pain is not well understood. Moreover, it should be noted that current therapies could hardly minimize neuropathic pain. Thus, it is of great importance to dissect the mechanism of LDH-induced neuropathic pain.

Chemokines belong to a family of proinflammatory cytokines, which contribute to cell growth, development, immune system regulation and inflammation ${ }^{4}$. In experimental studies, autologous nucleus pulposus (NP) implantation in the dorsal root ganglion (DRG) is used to initiate LDH in rats. Recent studies have reported that several chemokines were involved in the NP-induced neuropathic pain ${ }^{5-}$ ${ }^{6}$. For example, it has been reported that IL-8 mRNA expression was increased in the DRG after NP exposure while IL-8 inhibition could attenuated NP-induced allodynia in the spinal cord $^{6}$. Moreover, CCL2/CCR2 expression were also persistently increased in the dorsal root ganglion and spinal cord after NP implantation in rats ${ }^{5}$.

CX3CL1, also named as fractalkine, plays an important role in the regulation of chronic pain by binding with its preferred receptor CX3CR1. For example, it has been found that CX3CL1 expression was increased in spinal neurons and astrocytes after NP exposure ${ }^{7}$. Enhanced expression of CX3XL1 at mRNA and protein levels were reported both in the DRG and spinal cord (SC) in multiple sclerosis-induced neuropathic pain model ${ }^{8}$. However, the expression of CX3CL1 in the DRG in $\mathrm{LDH}$ rats are not studied; whether modification of CX3CL1 could attenuate LDH-induced neuropathic pain remains uninvestigated.

Nuclear factor-kappa B (NF-kB) is a transcription factor that has a pivotal function in the process of inflammation. It is well known that activated NF-KB could affect pain behavior via regulating inflammatory cytokines. Recent studies demonstrate that NF-KB decoys could suppresses cytokine expression in DRG and reduce hyperalgesia in both peripheral inflammatory pain model ${ }^{9}$ and rat $\mathrm{LDH}$ model[10]. However, whether NF-KB pathway is involved in CX3XL1 regulated $\mathrm{LDH}$-induced neuropathic pain is still unclear. In our study, we found that CX3XL1 in the DRG participated in LDH-induced neuropathic pain via NF-KB pathway.

\section{- Methods}

The Institutional Animal Care and Use Committee of Soochow University specifically approved this study.

Experiments were performed on adult male Sprague Dawley rats weighing 200-220g. Animals were housed in separated cages with free access to food and water at $24^{\circ} \mathrm{C}$ temperature and under a 12/12 hour light/dark cycle. All experimental and surgical procedures were carried out in accordance with the guidelines of the International Association for the Study of Pain ${ }^{11}$. All efforts were made to minimize animal suffering and to reduce the number of animals used.

\section{Disc herniation model}

Surgeries for the disc herniation model were performed as described in previous studies $^{10,12,13}$. In brief, rats were anesthetized with sodium pentobarbital $(50 \mathrm{mg} / \mathrm{kg}$ body weight, intraperitoneally) and placed in a 
prone position. Laminectomy was performed to expose the left $L 5$ nerve roots and DRG. The autologous NP was harvested from the C2-C3 intervertebral discs and was implanted next to the left $L 5$ nerve root just proximal to the DRG in the NP group. The rats in the sham group underwent the same surgical procedure except for implantation of NP. After surgery, the rats were housed in individual cages in the animal room until they fully recovered.

\section{Behavior test}

Rats were evaluated for mechanical allodynia and thermal hyperalgesia. The Behavior test was performed prior to surgery (day 0 ) and on days 1, 3, 5, 7, 10, 14, 21 and 28 after surgery. As described in previous study, mechanical thresholds were evaluated with the von Frey filaments (0.69-28.84g force; Stoelting, Wood Dale, IL) to calculate the $50 \%$ probability thresholds for mechanical paw withdrawal ${ }^{12}$. The rats left plantar surface of the hind paw, corresponding to the surgery, was stimulated in the L5 spinal nerve innervation area by the filaments, beginning with the $0.69 \mathrm{~g}$ filament. The filament was applied to the paw surface for about $3 \mathrm{~s}$ in a stepwise ascending or descending order following negative or positive withdrawal responses until six consecutive responses were noted.

Thermal hyperalgesia was measured by foot withdrawal latency to heat stimulation according to the previous studies, ${ }^{6,13}$. Briefly, each rat was placed in a Plexiglas box containing a smooth glass floor, the temperature maintaining at $28^{\circ} \mathrm{C}$. The heat source was provided by the analgesia meter (IITC Model 336 Analgesia Meter, Life Science) when measuring the latency to withdrawal from a thermal stimulus. The heat stimulus turned off manually when the hind paw moved (the maximum of $20 \mathrm{~s}$ to prevent tissue damage). Each rat was taken 5 tests of each hind paw, $30 \mathrm{~s}$ apart, at 10 mins intervals. A total mean data at each time point was calculated for each hindpaw ${ }^{14}$.

\section{Real-time PCR}

Total RNA was isolated from the left L5 DRG tissues at $0,1,5,10$, and 21 days after surgery. The protocol for real-time PCR was described as previous papers ${ }^{8}$. A SYBR green I kit was used to perform the PCR reaction following manufacturers protocols (Bio-Rad, Hercules, CA, USA). To amplify rat CX3CL1, the following primers were used: $C X 3 C L 1$ sense primer 5'-GAA TTC CTG GCG GGT CAG CAC CTC GGC ATA -3' and antisense primer 5' -AAG CTT TTA CAG GGC AGC GGT CTG GTG GT -3'; GAPDH sense primer 5'-ACC AGG GCT GCT TTT AAC TCT G-3' and antisense primer 5' - CCT TGA CTG TGC CGT GGA AC -3'. PCR was performed by 40 amplification cycles of denaturation at $95^{\circ} \mathrm{C}$ for 15 seconds and annealing at $60^{\circ} \mathrm{C}$ for 60 seconds. Expression levels were normalized to GAPDH.

\section{Western blot}

Western blot analysis was performed according to the manufacturer's protocol as previous reported ${ }^{15}$. L5 DRGs were immediately removed from deeply anesthetized rats and homogenized on ice in the lysis buffer (Cell Signaling, Danvers, MA). Equal amount of samples was run on a $10 \%$ SDS-polyacrylamide gel electrophoresis and transferred to a polyvinylidene difluoride membrane. After $1 \mathrm{~h}$ blocking in the block buffer (5\% nonfat dried milk), the membranes were incubated with the primary antibodies against CX3CL1 (1:500, R\&D Systerms, USA), NF-jB p65 (1:1000, Abcam, USA), phosphorylated NF-jB p65 (Ser311; 1:1000, CST, USA), and GAPDH (1:20000, Sigma, USA) at $4^{\circ} \mathrm{C}$ overnight. The membranes were then incubated with a horseradish peroxidaselabeled secondary antibody. ECL detection kit (Millipore, Billerica, MA, USA) were used to detect the immune complex. Protein expression was normalized against GAPDH. Western blot 
bands were quantified with the Quantity One image software (Bio-Rad).

\section{Statistical analysis}

All data were expressed as the meansSEM and analyzed using the Tukey post hoc test by SPSS 13.0 (SPSS, USA). $P$ values of less than 0.05 were considered significant.

\section{Results}

\section{Behavioral test}

All rats shown stable responses to mechanical stimulation before surgery. In the rats with $\mathrm{LDH}$, mechanical withdrawal threshold showed a significant decrease 1 day after the surgery to 28 days after the surgery, compared with the Blank group (rats without the surgery) and the Sham group (Rats underwent the surgery without implantation of NP ). There is not any significant difference in the mechanical withdrawal threshold between Blank group and Sham group (Figure 1A). Moreover, similar results were shown on the thermal withdrawal latency study. Compared to the Sham group, thermal withdrawal latency reduced markedly in the rats with LDH from 1day after the surgery and did not recover during the behavioral test (Figure 1B).
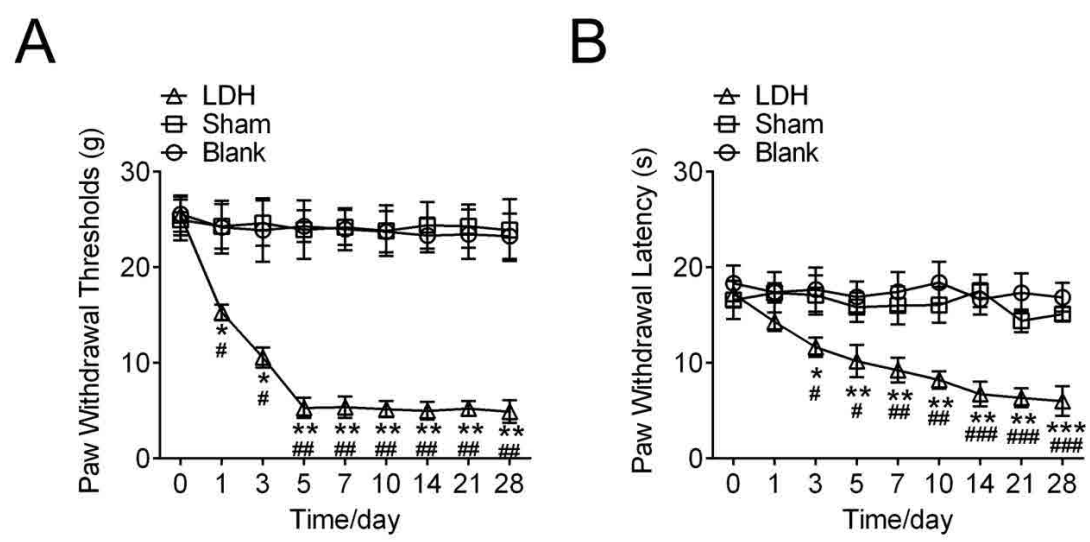

Figure 1 - Behavioral responses to mechanical stimulation of hind paw and heat in lumbar disc herniated models. Animals in lumbar disc herniated groups experienced significant mechanical allodynia (A) and thermal hyperalgesia (B) that started at day 1 and continued through day 28, compared with the Blank group and Sham group. Data are the means \pm SEM ( $n=10$ in each group, ${ }^{*} P<0.05,{ }^{*} P<0.01$, compared with Blank group, ${ }^{\#} P<0.05,{ }^{\#} P<0.01$, compared with Sham group ).

\section{Expression of $C X 3 X L 1$ to the $L D H$ rats in the} $D R G$

We then measured CX3CL1 mRNA expression in the DRG at 0, 1, 5, 10, 21 days after surgery by RT-PCR. The statistical results showed that the CX3CL1 mRNA level was significantly increased at 5 days after surgery $(P$
$<0.05)$ and peaked at 10 days after surgery $(P<$ 0.01) (Figure 2A). CX3CL1 mRNA level at 10 days after surgery was also significantly higher than that in the sham group $(P<0.01$; Figure $2 \mathrm{~A})$. Furthermore, western blot data demonstrated that CX3CL1 expression in the 5 and 10 days after surgery increased greatly comparing with Blank group ( $P<0.05$; Figure 2B). 
A

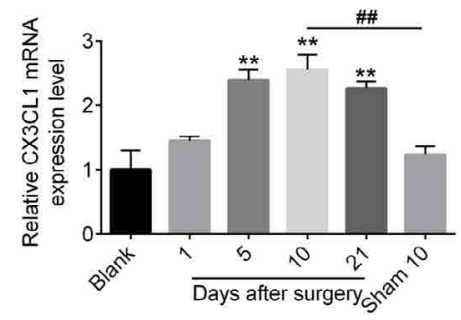

B

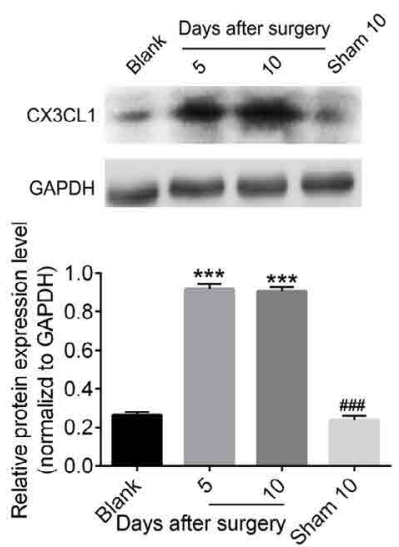

Figure 2 - Expression of CX3XL1 to the LDH rats in the DRG. DRG of LDH rats and control groups were collected for analysis in the presented time points. Representative histogram and blots shown the upregulation of CX3XL1 in the DRG of LDH rats, both in (A) mRNA level and (B) protein level. Data are the means \pm SEM ( $\mathrm{n}=5$ in each group, ${ }^{*} P<0.05,{ }^{* *} P<0.01,{ }^{* * *} P<0.001$, compared with Blank group, ${ }^{\#} P<0.05,{ }^{\# \#} P<0.01,{ }^{\# \#} P<0.001$, compared with LDH 10 day group ).

CX3CL1 downregulation attenuates $L D H$ Induced neuropathic pain

As both the RNA and protein expression of CX3CL1 was increased in DRG after LDH surgery, we speculated that CX3CL1 may contribute to the $L D H$-induced pain behaviors. Rats were consecutively intrathecal injected neutralizing antibody against CX3CL1 (10 $\mu \mathrm{g} / 10 \mu \mathrm{l}, \mathrm{R} \& \mathrm{D}$ system) or IgG antibody (10 $\mu \mathrm{g} / 10 \mu \mathrm{l}, \mathrm{R} \& \mathrm{D}$ system) as negative control for 10 days after LDH surgery and then the PWT and PWL were tested. The data in PWT and PWL test were found similar which showed that compared to the LDH group; continuous intrathecal injection of neutralizing antibody against CX3CL1 could attenuates thermal hyperalgesia induced by LDH model, whereas the IgG injection could not change paw withdrawal latency (Figure 3 A, B).

\section{A}

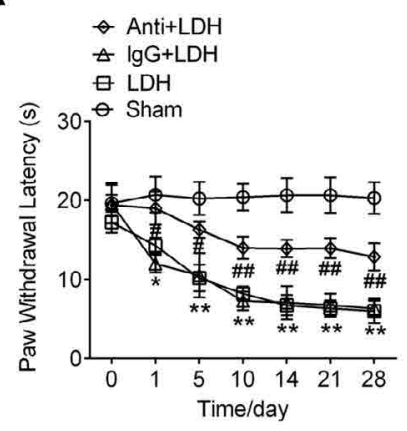

B

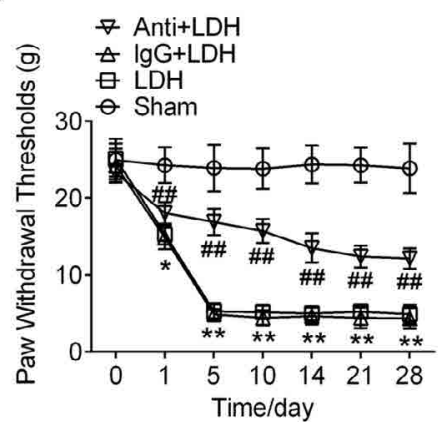

Figure 3 - Pre-treatment with CX3CL1 neutralizing antibody $(10 \mu \mathrm{g} / 10 \mu \mathrm{l}$, i.t.) but not $\operatorname{lgG}(10 \mu \mathrm{g} / 10 \mu \mathrm{l}$, i.t.) suppresses LDH-induced mechanical allodynia (A) and thermal hyperalgesia (B) Data are the means \pm SEM ( $n=10$ in each group, ${ }^{*} \mathrm{P}<0.05,{ }^{* *} \mathrm{P}<0.01,{ }^{* * *} \mathrm{P}<0.001$ compared with Sham group, ${ }^{\sharp} \mathrm{P}<0.05,{ }^{\#} \mathrm{P}<0.01,{ }^{, \# \# P} \mathrm{P}<0.001$ compared with LDH group).

\section{NF-KB activation in the DRGs of $L D H$ rats}

It is well-known that NF-kB contributes to neuropathic pain ${ }^{16}$. Here, we investigate whether NF-KB signaling is activated in our LDH model rats. We measured the expression of both phosphorylated and total NF-KB p65 in DRG at 5 and 10 days after surgery. 
The data demonstrated that the expression phosphorylated p65 (p-p65) at 5 and 10 days after the surgery was significantly increased in DRG compared with the Blank group without surgery. P-p65 value was not between the Blank group and the Sham group 10 days after the surgery. Moreover, the protein expression of total p65 were similar in each group (Figure 4).
A

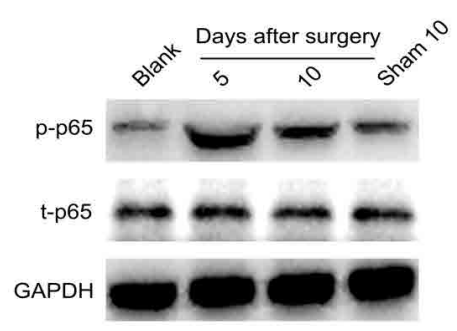

B

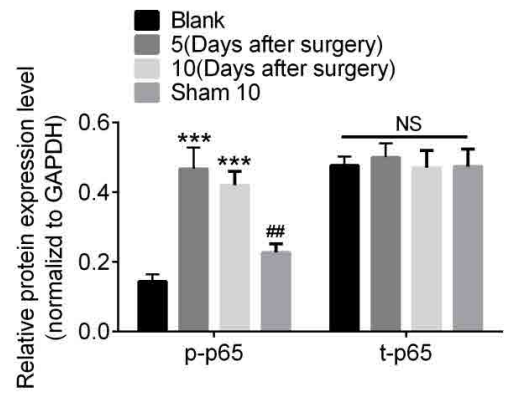

Figure 4 - NF-KB activation in the DRGs of LDH rats. DRG of LDH rats and control groups were collected for analysis in the presented time points. Representative histogram and blots shown the expression level of p-p65 and t-p65. Data are the means \pm SEM ( $n=5$ in each group, ${ }^{*} P<0.05,{ }^{*} P<0.01,{ }^{* * *} P<0.001$, compared with Blank group, ${ }^{\# P}<0.05,{ }^{\# \#} P<0.01$, compared with LDH 10 day group ).

Downregulation of NF-KBattenuates $L D H$ Induced neuropathic pain

Next, NF-kB p65 inhibitor PDTC (200 $\mathrm{ng} / 10 \mathrm{ml}$ for 10 days) were continuously intrathecal injected to the rats after the LDH surgery to downregulate the NF-KB activation. After the injection of PDTC, the expression of p-p65 was downregulated compared to the LDH group, which indicated the efficiency of p65 inhibitor PDTC (Figure 5A). Importantly, the data from behavior test PWT and PWL showed that continuous intrathecal injection of PDTC could attenuates thermal hyperalgesia induced by LDH model, compared to the LDH group (Figure $5 \mathrm{~B}, \mathrm{C}$ ).

A

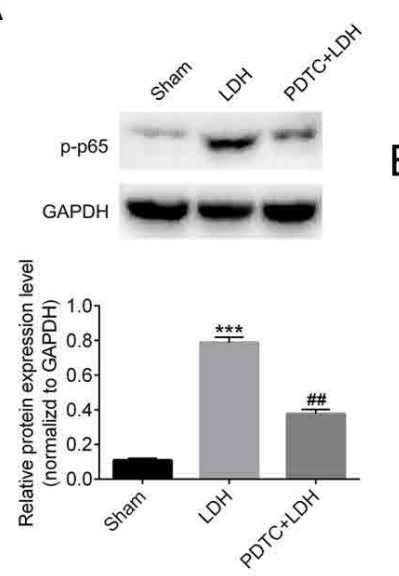

B

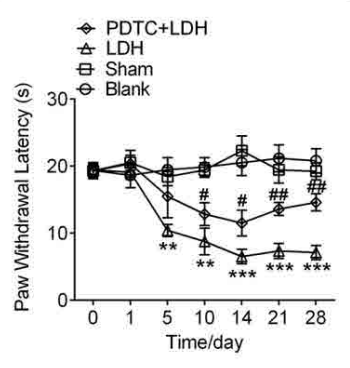

C

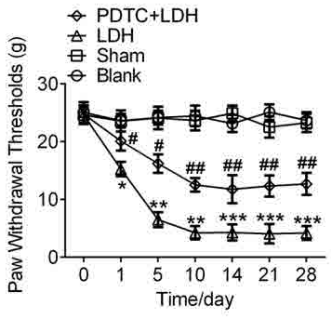

Figure 5 - Downregulation of NF-KB attenuates LDH-Induced neuropathic pain. LDH rats were injected with p65 inhibitor PDTC ( $200 \mathrm{ng} / 10 \mathrm{ml}$ for 10 days), DRG of LDH rats and control groups were collected for analysis. (A) Representative histogram and blots shown the decreased activation of p65. Data are the means \pm SEM ( $\mathrm{n}=5$ in each group, ${ }^{*} P<0.05,{ }^{*} P<0.01,{ }^{* *} P<0.001$, compared with Sham group, ${ }^{*} P<0.05,{ }^{*} P<0.01$, compared with $\mathrm{LDH}$ group ). (B, C) Intrathecal injection of PDTC obviously attenuated the mechanical allodynia and thermal hyperalgesia caused by LDH ( $\mathrm{n}=10$ in each group, ${ }^{*} P<0.05,{ }^{*} P<0.01,{ }^{* * *} P<0.001$ compared with Sham group, ${ }^{\#} P<0.05,{ }^{\# \#} P<0.01,{ }^{\# \#} P<0.001$ compared with LDH group ). 
$C X 3 X L 1$ regulates $L D H$-induced neuropathic painthrough NF-KB pathway

Knowing the fact that NF-KB pathway plays a cardinal role in regulating $\mathrm{LDH}$-induced neuropathic pain; we want to specifically study the function of CX3XL1 in the signaling pathway. We neutralized the CX3XL1 by injection antibody against it or IgG as control to the rats. It has been found that total p65 level were not affected too much, either in antibody group or IgG group when compared with blank or sham control. Consistently, the p-p65 level increased dramatically in LDH group. Interestingly, p-p65 was significantly inhibited in LDH rats injected with anti- CX3XL1 antibody; while the one with IgG antibody shown similar p-p65 level with LDH group (Figure 6). These data strongly suggests that $\mathrm{CX} 3 \mathrm{XL1}$ regulates $\mathrm{LDH}$-induced neuropathic pain by activating NF-KB pathway.
A

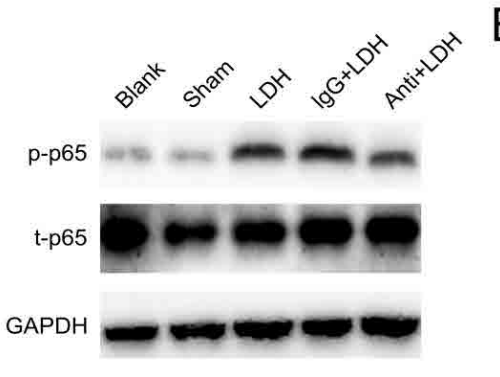

$B$

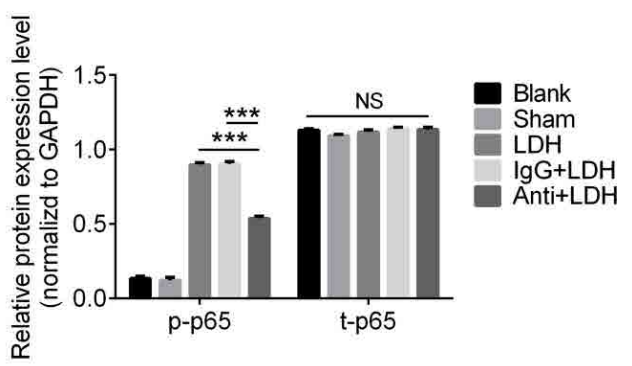

Figure 6 - CX3XL1 regulates LDH-induced neuropathic pain through NF-KB pathway. CX3CL1 neutralizing antibody $(10 \mu \mathrm{g} / 10 \mu \mathrm{l}$, i.t.) but not $\operatorname{lgG}(10 \mu \mathrm{g} / 10 \mu \mathrm{l}$, i.t.) reduces p-p65 level, while the total p65 level are not affected in each group. Representative histogram and blots shown the expression level of $p-p 65$ and p65 after antibody injection. Data are the means $\pm \operatorname{SEM}\left(n=5\right.$ in each group ${ }^{*} P<0.05,{ }^{* *} P<0.01,{ }^{* * *} P<0.001$, compared with Anti+LDH group).

\section{- Discussion}

Nowadays, $\mathrm{LDH}$ is accounting for $80 \%$ of patients that suffering from back pain ${ }^{17}$. However, the molecular mechanism underlying this disease is still not clear. A series of studies has used implantation of autologous NP in rats to mimic LDH pain, which were also adopted by $\mathrm{us}^{6,18}$. In this paper, we firstly reported the involvement of CX3XL1 in LDH-induced neuropathic pain. As its upregulation were identified in the DRG of LDH rats both by RTPCR and western blot. Moreover, inhibition of CX3XL1 activity by specific antibody injection significantly attenuated LDH-Induced neuropathic pain. Furthermore, we have found that NF-KB pathway was activated in this event. And using NF-kB p65 inhibitor PDTC also rescued the neuropathic pain induced by $\mathrm{LDH}$. More importantly, our data suggests that CX3XL1 was responsible for the activation of NF-KB pathway in LDH rat; which provides a new insight in LDH-induced neuropathic pain study.

It has been widely reported that neuroinflammation is one of the leading cause for neuropathic pain ${ }^{19}$. Chemokines, which belongs to the family of small cytokines, is the main mediator for the response of inflammation. They are divided into four subgroups according to their structures. CX3XL1, which characterized by its CX3C motif, is the only member of fourth chemokine group. There are two different forms of CX3XL1 exists in cells: one is membrane bounded and the other one is soluble form after cleavage in 
cytosol $^{20}$. By binding with its receptor CX3CR1, CX3XL1 is mainly found to be expressed in different types of neuronal cells or NK cells, T cells and smooth muscle cells ${ }^{21}$. Recent studies indicate that CX3XL1were found in some tumor cells, such as breast cancer cell and ovarian cancer cells ${ }^{22,23}$. However, the focus of CX3XL1 study is still on varieties of neurological disorders, such as peripheral neuropathy, experimental autoimmune encephalomyelitis and rheumatoid arthritis ${ }^{24}$. Our study is the first to report the involvement of CX3XL1 in $\mathrm{LDH}$ induced neuropathic pain.

NF-KB is a cardinal transcriptional factor that involved in the response of inflammation and cancers $^{25}$. Moreover, more studies have been done on NF-KB related to chronic pain. For instance, recent studies have shown its critical role in chemotherapy-induced chronic pain $^{26}$. We were specifically interested the molecular mechanism in $\mathrm{LDH}$ induced pain. Interestingly, NF-KB was activated in LDH rats and its activation was regulated by the expression of CX3XL1. For further studies, as it is well known that Akt, which is activated by phosphatidylinositol 3-kinase (PI3K), could phosphorylates NF-kB's inhibitor KB (IKB). This phosphorylation could leads to ubiquitination and degradation of $1 \mathrm{~KB}$; thus leading to the activation of NF-KB ${ }^{27}$. However, how this signaling pathway is regulated in $\mathrm{LDH}$ rats is still unclear, our further study will focus on PI3K-Akt signaling.

\section{Conclusions}

CX3XL1 could regulate $\mathrm{LDH}$-induced neuropathic pain through NF-kB pathway. Targeting CX3CL1 and NF-KB may represent a potential treatment for neuropathic pain caused by LDH.

\section{References}

1. Mulleman D, Mammou S, Griffoul I,
Watier $\mathrm{H}$, Goupille P. Pathophysiology of disk-related sciatica. I.--Evidence supporting a chemical component. Joint Bone Spine. 2006;73(2):151-8. doi: 10.1016/j. jbspin.2005.03.003.

2. Olmarker K, Brisby H, Yabuki S, Nordborg C, Rydevik B. The effects of normal, frozen, and hyaluronidase-digested nucleus pulposus on nerve root structure and function. Spine (Phila Pa 1976). 1997;22(5):471-5; discussion 476. PMID: 9076877.

3. Olmarker K, Rydevik B, Nordborg C. Autologous nucleus pulposus induces neurophysiologic and histologic changes in porcine cauda equina nerve roots. Spine (Phila Pa 1976). 1993;18(11):1425-32. PMID: 8235812.

4. Rollins BJ. Chemokines. Blood. 1997;90(3):909-28. PMID: 9242519.

5. Zhu X, Cao S, Zhu MD, Liu JQ, Chen JJ, Gao YJ. Contribution of chemokine CCL2/ CCR2 signaling in the dorsal root ganglion and spinal cord to the maintenance of neuropathic pain in a rat model of lumbar disc herniation. J Pain. 2014;15(5):516-26. doi: 10.1016/j.jpain.2014.01.492.

6. Kim SJ, Park SM, Cho YW, Jung YJ, Lee DG, Jang SH, Park HW, Hwang SJ, Ahn SH. Changes in expression of mRNA for interleukin-8 and effects of interleukin-8 receptor inhibitor in the spinal dorsal horn in a rat model of lumbar disc herniation. Spine (Phila Pa 1976). 2011;36(25):2139-46. doi: 10.1097/ BRS.0b013e31821945a3.

7. Park HW, Ahn SH, Kim SJ, Seo JM, Cho YW, Jang SH, Hwang SJ, Kwak SY. Changes in spinal cord expression of fractalkine and its receptor in a rat model of disc herniation by autologous nucleus pulposus. Spine (Phila Pa 1976). 2011;36(12):E753-60. doi: 10.1097/BRS.0b013e3181ef610b.

8. Zhu W, Acosta C, MacNeil B, Cortes C, Intrater $H$, Gong $Y$, Namaka M. Elevated expression of fractalkine (CX3CL1) and fractalkine receptor (CX3CR1) in the dorsal root ganglia and spinal cord in experimental autoimmune encephalomyelitis: implications in multiple sclerosis-induced neuropathic pain. Biomed Res Int. 2013;2013:480702. doi: 10.1155/2013/480702.

9. Sakaue G, Shimaoka M, Fukuoka T, Hiroi T, Inoue T, Hashimoto N, Sakaguchi T, Sawa Y, 
Morishita R, Kiyono H, Noguchi K, Mashimo T. NF-kappa B decoy suppresses cytokine expression and thermal hyperalgesia in a rat neuropathic pain model. Neuroreport. 2001;12(10):2079-84. PMID: 11447311.

10.Suzuki M, Inoue G, Gemba T, Watanabe T, Ito T, Koshi T, Yamauchi K, Yamashita M, Orita S, Eguchi Y, Ochiai N, Kishida S, Takaso M, Aoki Y, Takahashi K, Ohtori S. Nuclear factorkappa B decoy suppresses nerve injury and improves mechanical allodynia and thermal hyperalgesia in a rat lumbar disc herniation model. Eur Spine J. 2009;18(7):1001-7. doi: 10.1007/s00586-009-0940-x.

11.Zimmermann $M$. Ethical guidelines for investigations of experimental pain in conscious animals. Pain. 1983;16(2):109110. PMID: 6877845.

12.Kato K, Kikuchi S, Konno S, Sekiguchi M. Participation of 5-hydroxytryptamine in pain-related behavior induced by nucleus pulposus applied on the nerve root in rats. Spine (Phila Pa 1976). 2008;33(12):1330-6. doi: 10.1097/BRS.0b013e318173298b.

13.Zhang XS, Li X, Luo HJ, Huang ZX, Liu CC, Wan Q, Xu SW, Wu SL, Ke SJ, Ma C. Activation of the RAGE/STAT3 pathway in the dorsal root ganglion contributes to the persistent pain hypersensitivity induced by lumbar disc herniation. Pain Physician. 2017;20(5):41927. PMID: 28727705.

14.Dirig DM, Salami A, Rathbun ML, Ozaki GT, Yaksh TL. Characterization of variables defining hindpaw withdrawal latency evoked by radiant thermal stimuli. J Neurosci Methods. 1997;76(2):183-91. PMID: 9350970.

15.Kato K, Sekiguchi M, Kikuchi S, Konno S. The effect of a 5-HT2A receptor antagonist on pain-related behavior, endogenous 5-hydroxytryptamine production, and the expression 5-HT2A receptors in dorsal root ganglia in a rat lumbar disc herniation model. Spine (Phila Pa 1976). 2015;40(6):357-62. doi: $10.1097 /$ brs.0000000000000769.

16.Lee KM, Kang BS, Lee HL, Son SJ, Hwang $\mathrm{SH}$, Kim DS, Park JS, Cho HJ. Spinal NF$\mathrm{kB}$ activation induces COX-2 upregulation and contributes to inflammatory pain hypersensitivity. Eur J Neurosci. 2004;19(12):3375-81. doi: 10.1111/j.0953816X.2004.03441.x.
17.Andersson GB. Epidemiological features of chronic low-back pain. Lancet. 1999;354(9178):581-5. doi: 10.1016/s01406736(99)01312-4.

18.Wang Q, Zhu H, Zou K, Yuan B, Zhou YL, Jiang X, Yan J, Xu GY. Sensitization of P2X3 receptors by cystathionine beta-synthetase mediates persistent pain hypersensitivity in a rat model of lumbar disc herniation. Mol Pain. 2015;11:15. doi: 10.1186/s12990-0150012-7.

19.Sekiguchi M, Sekiguchi Y, Konno S, Kobayashi $\mathrm{H}$, Homma Y, Kikuchi S. Comparison of neuropathic pain and neuronal apoptosis following nerve root or spinal nerve compression. Eur Spine J. 2009;18(12):197885. doi: 10.1007/s00586-009-1064-z.

20.Bazan JF, Bacon KB, Hardiman G, Wang W, Soo K, Rossi D, Greaves DR, Zlotnik A, Schall TJ. A new class of membranebound chemokine with a CX3C motif. Nature. 1997;385(6617):640-4. doi: 10.1038/385640a0.

21.Lindia JA, McGowan E, Jochnowitz N, Abbadie C. Induction of CX3CL1 expression in astrocytes and CX3CR1 in microglia in the spinal cord of a rat model of neuropathic pain. J Pain. 2005;6(7):434-8. doi: 10.1016/j. jpain.2005.02.001.

22.Geller MA, Bui-Nguyen TM, Rogers LM, Ramakrishnan S. Chemotherapy induces macrophage chemoattractant protein-1 production in ovarian cancer. Int J Gynecol Cancer. 2010;20(6):918-25. doi: 10.1111/ IGC.0b013e3181e5c442.

23. Kim YS, An HT, Kim J, Ko J. Effects of protein kinase Cdelta and phospholipase C-gamma1 on monocyte chemoattractant protein-1 expression in taxol-induced breast cancer cell death. Int J Mol Med. 2009;24(6):853-8. PMID: 19885629.

24.Umehara H, Bloom ET, Okazaki T, Nagano $\mathrm{Y}$, Yoshie O, Imai T. Fractalkine in vascular biology: from basic research to clinical disease. Arterioscler Thromb Vasc Biol. 2004;24(1):34-40. doi: 10.1161/01. atv.0000095360.62479.1f.

25.Vlahopoulos SA, Cen O, Hengen N, Agan J, Moschovi $M$, Critselis $E$, Adamaki $M$, Bacopoulou F, Copland JA, Boldogh I, Karin $M$, Chrousos GP. Dynamic aberrant NFkappaB spurs tumorigenesis: a new model 
encompassing the microenvironment. Cytokine Growth Factor Rev. 2015;26(4):389-403. doi: 10.1016/j. cytogfr.2015.06.001.

26.Huang ZZ, Li D, Ou-Yang HD, Liu CC, Liu XG, Ma C, Wei JY, Liu Y, Xin WJ. Cerebrospinal fluid oxaliplatin contributes to the acute pain induced by systemic administration of oxaliplatin. Anesthesiology. 2016;124(5):1109-21. doi: 10.1097/ aln.0000000000001084.

27.Bai D, Ueno L, Vogt PK. Akt-mediated regulation of NFkappaB and the essentialness of NFkappaB for the oncogenicity of PI3K and Akt. Int J Cancer. 2009;125(12):286370. doi: 10.1002/ijc. 24748 .

\section{Correspondence:}

Chenglong Liu

Department of Anaesthesiology

Gaoyou People's Hospital, Gaoyou Hospital

Affiliated

Soochow University

Yangzhou, Jiangsu 225600 China

Received: Mar 28, 2018

Review: May 24, 2018

Accepted: June 22, 2018
Conflict of interest: none

Financial source: none
${ }^{1}$ Research performed at Department of Anaesthesiology, Gaoyou People's Hospital, Gaoyou Hospital Affiliated, Soochow University, China. 